A\&L

ISSN 2709-0205

Vol 2 Issue 3 (2021)

https://doi.org/10.47855/jal9020-2021-3

\title{
Estimating biological age by hematological blood parameters
}

\author{
Anatoly V. Pisaruk, Ludmila V. Mekhova \\ D. F. Chebotarev Institute of Gerontology NAMS of Ukraine, Kyiv, Ukraine \\ https://doi.org/10.47855/ja19020-2021-3-2
}

Correspondence: avpisaruk54@gmail.com

Received: 15.09.2021; Accepted: 07.10.2021; Published: 07.10.2021

\begin{abstract}
For the estimation of the biological age (BA) of people based on hematological parameters of the clinical blood test there were used MLR and Deep Neural Networks. In the archive of the Institute of Gerontology NAMS of Ukraine there were selected people aged from 20 up to 90 years (440 men and 504 women), who had all hematological parameters within normal limits. When using the MLR method, the multiple correlation coefficients $(\mathrm{R})$ have low values for both men (0.37) and women (0.38). The use of Deep Neural Networks has given good results. The values of the correlation coefficients between BA and chronological age were 0.92 for men and 0.79 for women. The average absolute error in determining BA was 3.68 years for the men and 6.55 years for the women. The developed method for assessing hematological age can be used in clinical practice to identify people with the risk of developing hematological pathology, as well as in population researches.
\end{abstract}

Keywords: biological age, hematological blood parameters, deep neural network

The variations in the rate of aging for the different people have conditioned a necessity of the more accurate assessment of it than it was given by chronological age (CA). For this, the calculation of biological age (BA) is used $[1,2]$. Recently, many studies have been appeared where the data from clinical blood tests are used to assess human BA [3-6]. This approach has advantages due to the availability and standardization of the tests. A huge array of data of the clinical blood parameters among the people of different ages has been accumulated (Big Data). However, the age-related changes in many clinical blood parameters are insignificant. This makes them difficult to use as biomarkers of aging. The use of the multiple regression equation for the construction of the BA formula does not provide sufficient accuracy of the estimation. Recently, a new BA assessment method has been appeared based on the use of Deep Neural Networks [7-9]. This method has shown high accuracy in recognizing a person's age. This is achieved through the use of a large number of indicators and the Deep learning algorithm. Previously, we have successfully used this approach to determine the age of a person by biochemical blood tests [10]. In the presented research, we used Deep Neural Networks to assess BA of humans based on hematological parameters of clinical blood test.

\section{Materials and methods}

The data from the archive of the Institute of Gerontology NAMS of Ukraine were used. We have selected people aged from 20 up to 90 years (440 men and 504 women), whose all hematological parameters were within the normal range. The average age of men was $64.8 \pm 13.2$, women $-61.9 \pm 14.1$ years. The 
concentration of blood cells was determined using a hematological analyzer. Hematocrit, thrombokrit, erythrocyte and platelet indices were calculated: MCV - Mean Corpuscular Volume;

$\mathrm{MCH}$ - Mean Content of Hemoglobin in the erythrocyte;

MCHC - Mean Corpuscular Hemoglobin Concentration;

RDW - Red cell Distribution Width;

MPV - Mean Platelet Volume;

PDW - relative Width of Platelet Distribution by volume;

MCV - Mean Corpuscular Volume.

The erythrocyte sedimentation rate (ESR) was measured using a standard method.

Multiple linear regression (MLR) and Neural Networks methods were used to analyze the obtained data. We had used Neural Networks algorithms of different structures, that were presented in the Statistica 7 program. They were trained to recognize a person's age by hematological blood parameters, and the accuracy of the obtained results was evaluated. It was chosen the structure of the neural network, which provided the best result - the highest correlation coefficient of the predicted age with the actual one, as well as the minimal error in the determining of the age.

Statistical processing of the obtained data was performed using the Statistica 7 software (StatSoft, USA). Standard statistical procedures were used, including variational and regression analyzes, as well as neural network analysis (Neural Networks).

\section{Results and discussion}

Table 1 shows the average meanings and standard errors (SE) of hematological parameters in men and women. It can be seen that almost all indicators have significant differences by gender. Therefore, a separate data analysis is required for each gender.

Table 1

Mean values and standard errors (SE) of hematological parameters in men and women (Marked correlations are significant at $\mathrm{p}<0.05$ )

\begin{tabular}{|l|r|r|r|r|c|}
\hline \multirow{2}{*}{\multicolumn{1}{|c|}{ Indicators }} & \multicolumn{2}{|c|}{ Male } & \multicolumn{2}{c|}{ Female } & \multirow{2}{*}{$p$} \\
\cline { 2 - 5 } & Mean & \multicolumn{1}{c|}{ SE } & Mean & \multicolumn{1}{c|}{ SE } & \\
\hline Leukocytes, 10\%/L & 6.525 & 0.098 & 6.271 & 0.074 & 0.035530 \\
\hline Erythrocytes, 1012/L & 4.630 & 0.024 & 4.486 & 0.017 & 0.000001 \\
\hline Hemoglobin, g/L & 139.1 & 0.695 & 131.2 & 0.511 & 0.000000 \\
\hline Hematocrit & 0.413 & 0.002 & 0.390 & 0.002 & 0.000000 \\
\hline Platelets, 10 /L & 223.3 & 2.683 & 238.8 & 2.382 & 0.000021 \\
\hline Thrombokrit & 0.193 & 0.002 & 0.206 & 0.003 & 0.000635 \\
\hline MCV, fL & 89.46 & 0.270 & 87.24 & 0.312 & 0.000000 \\
\hline MCH, pg & 30.07 & 0.109 & 29.29 & 0.101 & 0.000000 \\
\hline MCHC, g/L & 336.7 & 0.772 & 336.9 & 0.662 & 0.847845 \\
\hline RDW, fL & 13.54 & 0.045 & 13.53 & 0.056 & 0.972056 \\
\hline MPV, fL & 8.640 & 0.040 & 8.582 & 0.034 & 0.273231 \\
\hline PDW, \% & 13.62 & 0.086 & 13.84 & 0.077 & 0.061512 \\
\hline Lymphocytes, \% & 31.28 & 0.399 & 31.63 & 0.342 & 0.504355 \\
\hline Monocytes, \% & 5.826 & 0.098 & 5.552 & 0.094 & 0.047948 \\
\hline Granulocytes, \% & 62.86 & 0.416 & 62.68 & 0.371 & 0.755942 \\
\hline ESR, mm/hr & 12.81 & 0.411 & 15.19 & 0.400 & 0.000049 \\
\hline
\end{tabular}


Table 2 shows the meanings of the correlation coefficients (Pearson) of hematological parameters with age for men and women. There are not many reliable correlations. Correlations with age of ESR and concentration of platelets in blood are common for both genders. At the same time, ESR increases with age, and the concentration of platelets decreases. ESR has the highest positive correlation with age ( 0.27 for the men and 0.29 for the women). In men, the concentration of erythrocytes and hemoglobin negatively correlates with age, which indicates a slowdown of erythropoiesis. There is no such correlation in women. This is due to the fact that in the reproductive period, women have regular menstrual blood loss and their average level of erythrocytes and hemoglobin is lower than in men (Tab.1). In men, RDW significantly increases with age, in women, MCHC decreases. The increase in RDW with age is connected with an increase in the proportion of old red blood cells, that have a larger volume.

\section{Pearson correlation coefficients between chronological age and biomarkers (Marked correlations are significant at $\mathrm{p}<0.05$ )}

Table 2

\begin{tabular}{|l|r|r|}
\hline \multicolumn{1}{|c|}{ Indicators } & \multicolumn{1}{c|}{ Male } & \multicolumn{1}{c|}{ Female } \\
\hline Leukocytes & -0.01 & -0.08 \\
\hline Erythrocytes & -0.08 & -0.04 \\
\hline Hemoglobin & -0.12 & -0.05 \\
\hline Hematocrit & -0.11 & -0.02 \\
\hline Platelets & -0.11 & -0.13 \\
\hline Thrombokrit & -0.09 & -0.05 \\
\hline MCV & -0.07 & 0.05 \\
\hline MCH & -0.02 & -0.04 \\
\hline MCHC & 0.17 & 0.07 \\
\hline RDW & 0.00 & -0.04 \\
\hline MPV & 0.00 & 0.03 \\
\hline PDW & 0.04 & -0.07 \\
\hline Lymphocytes & 0.04 & 0.10 \\
\hline Monocytes & -0.06 & 0.03 \\
\hline Granulocytes & 0.27 & 0.29 \\
\hline ESR & & \\
\hline
\end{tabular}

For the construction of the model of hematological age, the MLR method with sequential inclusion of indicators was used. It can be seen that the coefficients of the multiple correlation and determination ( $R$ and $R 2$ ) have low values for both men $(0.37 ; 0.12)$ and women $(0.38 ; 0.14)$.

As a result, the standard error of calculating of the age is big (12 years for men and 13 years for women). The accuracy of calculating of the age is insufficient for using MLR models in practice. 


\section{Regression summary for dependent variable: Age (Male) \\ (Marked correlations are significant at $\mathrm{p}<0.05$ )}

$R=0.368 ; R^{2}=0.136 ;$ Adjusted $R^{2}=0.118 ; F(8.440)=7.781 p<0.00001 ;$ Std. Error of estimate: 12.397

\begin{tabular}{lrrrrrr}
\multicolumn{1}{c}{ Beta } & Std. Err. & \multicolumn{1}{c}{ B } & Std.Err. & t & p-level \\
\multicolumn{1}{c}{ Intercept } & & & 70.7382 & 18.6741 & 3.7880 & 0.0002 \\
ESR & 0.3166 & 0.0480 & 0.5135 & 0.0779 & 6.5955 & 0.0000 \\
Platelets & -0.2928 & 0.1173 & -0.0719 & 0.0288 & -2.4961 & 0.0130 \\
RDW & 0.1193 & 0.0482 & 1.7254 & 0.6979 & 2.4724 & 0.0138 \\
Lymphocytes & 0.1224 & 0.0496 & 0.2016 & 0.0818 & 2.4651 & 0.0141 \\
MPV & -0.1175 & 0.0613 & -1.9073 & 0.9950 & -1.9170 & 0.0560 \\
MCHC & -0.0653 & 0.0488 & -0.0557 & 0.0416 & -1.3372 & 0.1819 \\
Leukocytes & 0.0527 & 0.0501 & 0.3546 & 0.3372 & 1.0517 & 0.2936 \\
Thrombokrit & 0.1209 & 0.1198 & 34.8906 & 34.5511 & 1.0098 & 0.3132
\end{tabular}

Note: $\mathrm{R}$ - correlation coefficient of indicators with the model; $\mathrm{R}^{2}$ - coefficient of model determination; Adjusted $\mathrm{R}^{2}$ - adjusted R-square (taking into account the number of predictors in the model); F - Fisher's test; $\mathrm{t}$ - Student's test; $\mathrm{p}$ - assessment of the significance of the model; SE of estimate - standard error of estimation; Intercept - free member of the equation; $b$ - regression coefficient; $b^{*}-$ standardized regression coefficient; SE of $b^{*}$ - standardized error of the coefficient.

Regression summary for dependent variable: Age (Female)

Table 4

(Marked correlations are significant at $\mathrm{p}<0.05$ )

$R=0.380 ; R^{2}=0.144$ Adjusted $R^{2}=0.127 ; F(10.504)=8.512 ; p<0.00001 ;$ Std. Error of estimate: 13.067

$\begin{array}{lllll}\text { Beta Std. Err. } & \text { B } & \text { Std.Err. } & \mathbf{t} & \text { p-level }\end{array}$

Intercept

\begin{tabular}{|c|c|c|c|c|c|c|}
\hline Intercept & & & 72.5780 & 25.5700 & 2.8384 & 0.0047 \\
\hline ESR & 0.3121 & 0.0454 & 0.4815 & 0.0701 & 6.8711 & 0.0000 \\
\hline Platelets & -0.2185 & 0.0558 & -0.0562 & 0.0144 & -3.9149 & 0.0001 \\
\hline MPV & -0.0837 & 0.0464 & -1.5891 & 0.8807 & -1.8044 & 0.0718 \\
\hline RDW & 0.1101 & 0.0459 & 1.2375 & 0.5161 & 2.3977 & 0.0169 \\
\hline Hematocrit & 0.0611 & 0.0472 & 24.6413 & 19.0666 & 1.2924 & 0.1968 \\
\hline Leukocytes & -0.0720 & 0.0481 & -0.5932 & 0.3963 & -1.4968 & 0.1351 \\
\hline Lymphocytes & -0.1830 & 0.1219 & -0.3314 & 0.2208 & -1.5010 & 0.1340 \\
\hline Thrombokrit & 0.0615 & 0.0477 & 13.9881 & 10.8314 & 1.2914 & 0.1971 \\
\hline Granulocytes & -0.1310 & 0.1230 & -0.2211 & 0.2076 & -1.0651 & 0.2874 \\
\hline $\mathrm{MCV}$ & 0.0472 & 0.0460 & 0.0926 & 0.0902 & 1.0270 & 0.3049 \\
\hline
\end{tabular}

Note: the same as in the table 3. 


\section{Neural network analysis.}

Using a neural network simulated in the program Statistica, the problem of recognizing of the age of the examined people by hematological blood parameters was solved. To train the neural network, the results of the research of men and women, as well as their CA, were submitted to its input. CA has been defined as an output variable of continuous type. During the process of training, the network is looking for dependencies between input and output data by changing the weighting coefficients of connections between neurons. The obtained best structure of the network was different for men and women. For the men, the MLP neural network with 16 input variables and 3 inner layers of 16, 8, 4 neurons showed the best results (Fig. 1). For the women, this network has 3 inner layers of 16, 10, and 8 neurons (Fig. 2).

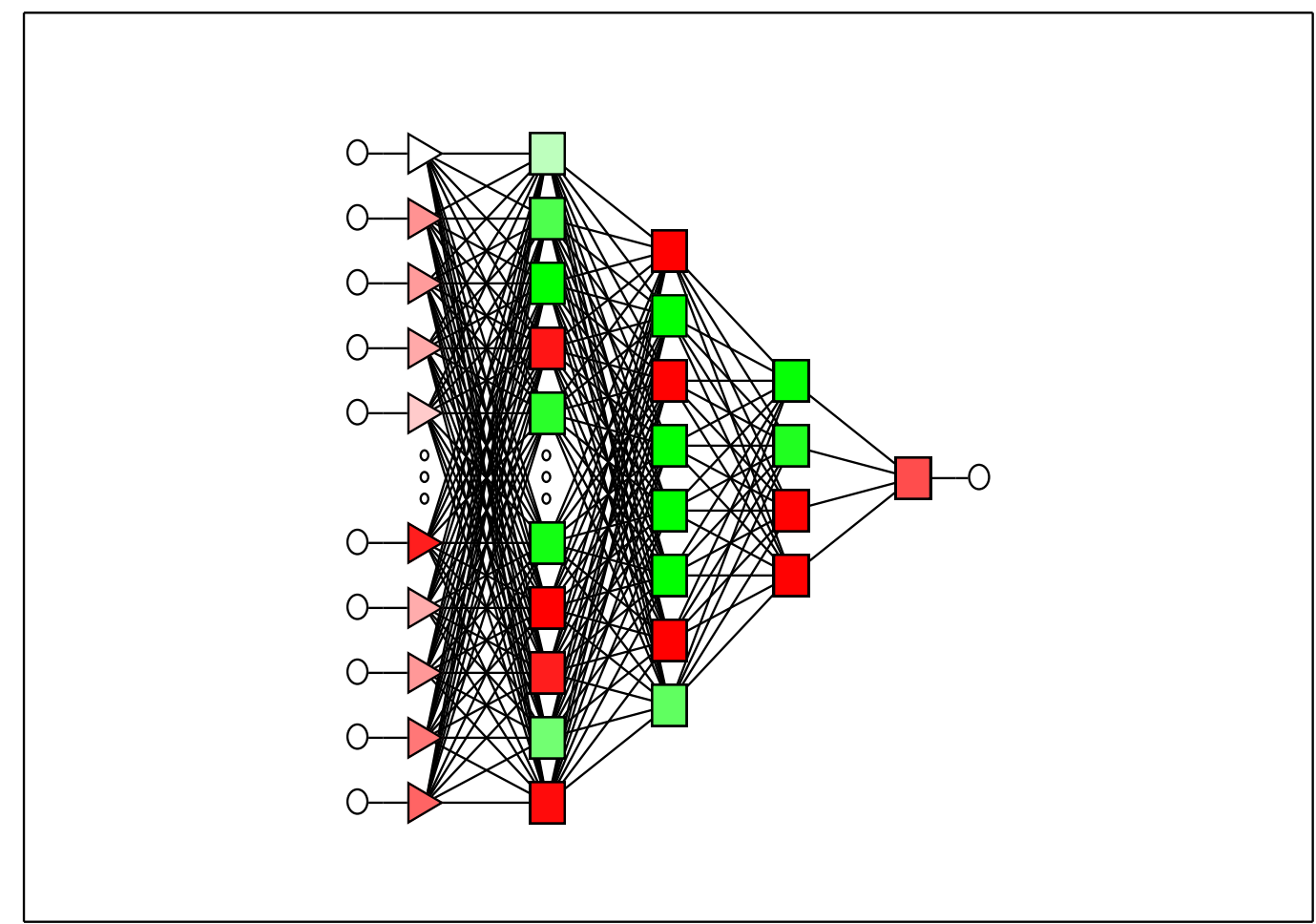

Figure 1. The structure of a neural network for calculating the biological age of men. 


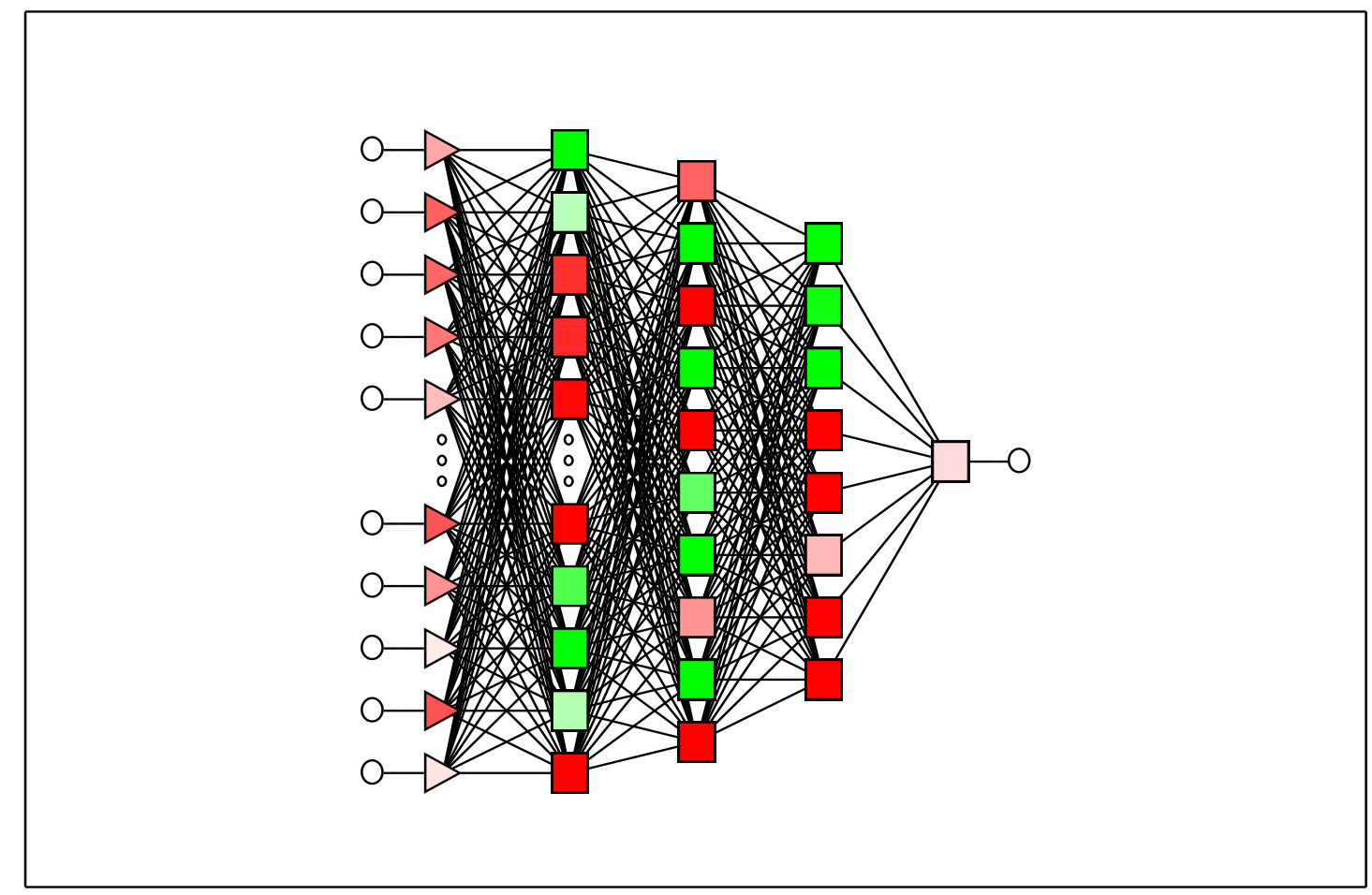

Figure 2. The structure of a neural network for calculating the biological age of women.

The results of calculating of the age of people with a neural network are shown in figures 3-4, where the BA and CA ratios are shown. The values of the correlation coefficients between BA and CA were 0.92 for the men and 0.79 for the women. This is a very good result.

The magnitude of the difference between BA and CA was calculated for each person. The average absolute error in determining BA was 3,68 years for the men and 6,55 years for the women.

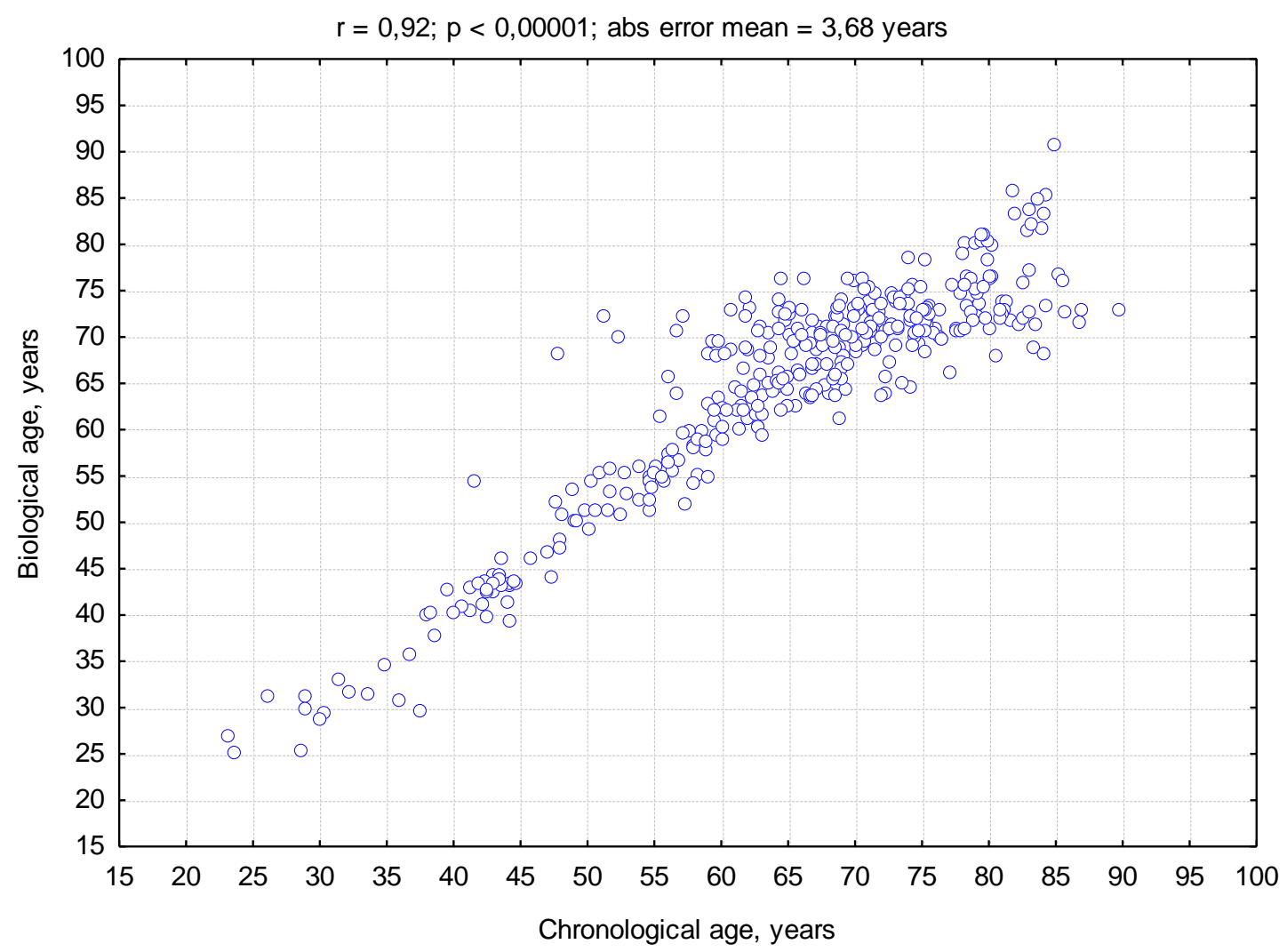

Figure 3. Correlation of chronological and biological age of men. 


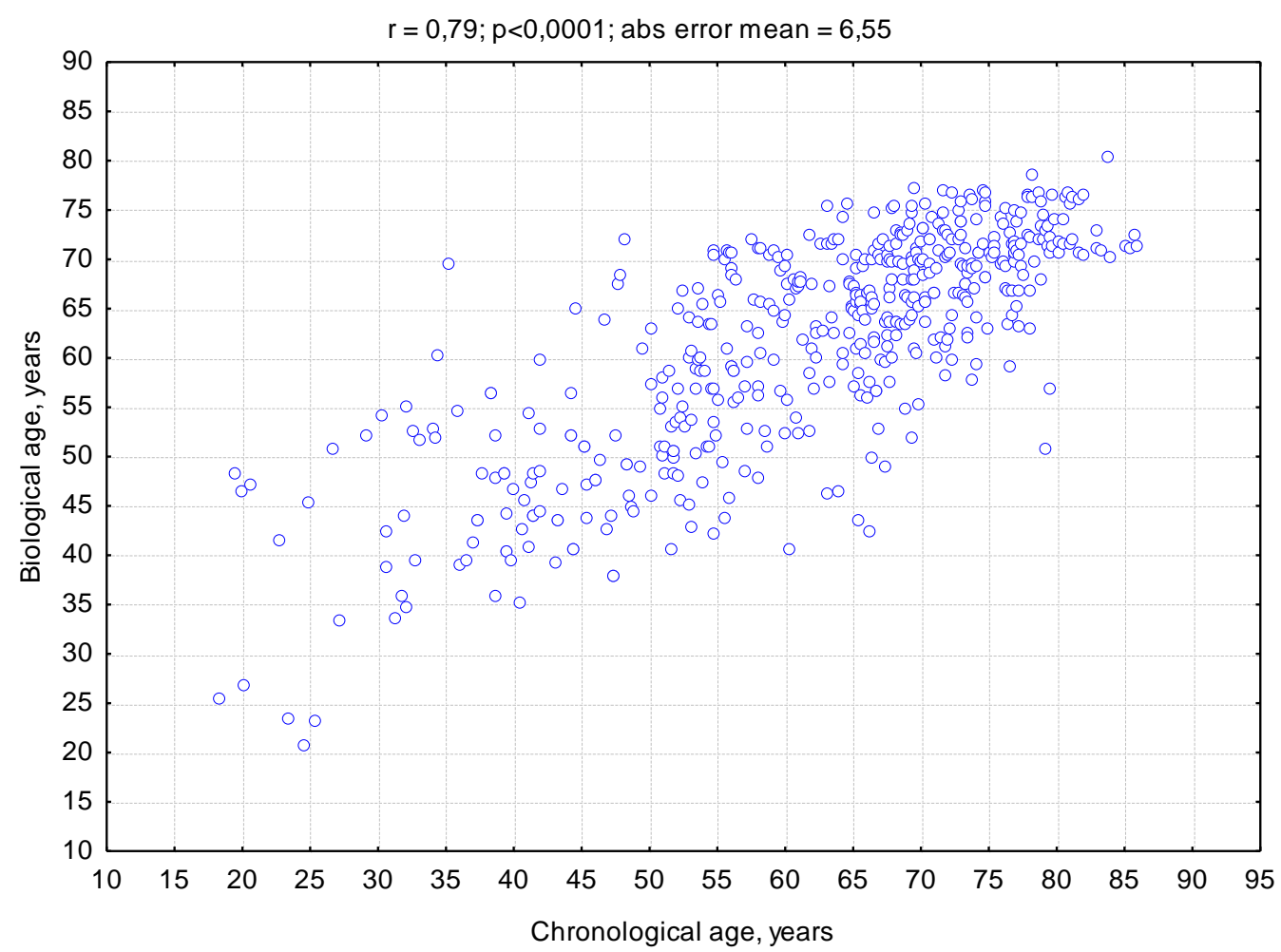

Figure 4. Correlation of chronological and biological age of women.

Comparing the two approaches that we had used for assessing BA based on the data for determining hematological parameters, the following can be noted. The MLR method did not provide a satisfactory assessment of BA for the men and the women. The use of a trained neural network provides good accuracy of BA assessment, but requires the use of a PC with Statistica program installed or the development of special software.

BA, defined by hematological parameters, is the BA of the hematopoietic system and can be used in clinical practice to identify people with the risk of developing of hematological pathology, as well as in population studies.

Author Contributions: All authors participated equally in writing this article.

Conflicts of Interest: The authors declare no conflict of interest.

Information about Authors:

Anatoly V. Pisaruk - DSc (Medicine), Head of the Laboratory for Mathematical Modeling of Aging Processes; https://orcid.org/0000-0002-6832-8614

Ludmila V. Mekhova - PhD (Medicine), Senior Researcher of the Laboratory for Mathematical Modeling of Aging Processes; https://orcid.org/0000-0002-8445-179 


\section{References}

1. Murabito, J. M.; Zhao, Q.; Larson, M. G.; Rong, J.; Lin, H. et al. Measures of biologic age in a community sample predict mortality and age-related disease: the framingham offspring study. J Gerontol Ser A Biol Sci Med Sci 2018, 73, 757-762. doi: 10.1093/gerona/glx144

2. Jia, L.; Zhang, W.; Chen, X. Common methods of biological age estimation. Clin Interv Aging 2017, 12, 759-772. doi: 10.2147/CIA.S134921.

3. Mamoshina, P.; Kochetov, K.; Putin, E.; Cortese, F.; Aliper, A.et al. Population specific biomarkers of human aging: a big data study using South Korean, Canadian and Eastern European patient populations. J Gerontol Ser A 2018, 1, 1-9. doi: 10.1093/gerona/gly005

4. Mamoshina, P.; Vieira, A.; Putin, E.; Zhavoronkov, A. Applications of deep learning in biomedicine. Mol Pharm 2016, 13, 1445-1454. doi: 10.1021/acs.molpharmaceut.5b00982

5. Sebastiani, P.; Thyagarajan, B.; Sun, F.; Schupf, N.; Newman et al. Biomarker signatures of aging. Aging Cell 2017, 16, 329-338. doi: 10.1111/acel.12557

6. Putin, E.; Mamoshina, P.; Aliper, A.; Korzinkin, M.; Moskalev, A. Deep biomarkers of human aging: application of deep neural networks to biomarker development. Aging (Albany NY) 2016, 8, 1021-1033. doi: 10.18632/aging.100968

7. Caballero, F.F.; Soulis, G.; Engchuan, W.; Sanchez-Niubo, A.; Arndt, H. et al. Advanced analytical methodologies for measuring healthy ageing and its determinants, using factor analysis and machine learning techniques: the ATHLOS project. Sci Rep 2017, 7, 439-455. doi: 10.1038/srep43955.7

8. Ching, T.; Himmelstein, D. S.; Beaulieu-Jones, B. K.; Kalinin, A. A.; Do B. T. et al. Opportunities and obstacles for deep learning in biology and medicine. J R Soc Interface 2018, 15. doi: 10.1098/rsif.2017.0387

9. Cole, J. H.; Poudel, R. P. K.; Tsagkrasoulis, D.; Caan, M. W. A. et al. Predicting brain age with deep learning from raw imaging data results in a reliable and heritable biomarker. Neuroimage 2017, 163, 115124. doi: 10.1016/j.neuroimage.2017.07.059

10. Korkushko, O. V.; Pisaruk, A. V.; Chyzhova,V. P. Estimation of human metabolic age using regression and neural network analysis. Zaporozhye medical journal 2021, 23, 60-64. doi:

10.14739/2310-1210.2021.1.224883 